Service social

\title{
Antinomie ou concordance des perspectives théoriques en service social?
}

\section{André Beaudoin}

Volume 42, numéro 3, 1993

Perspectives théoriques

URI : https://id.erudit.org/iderudit/706627ar

DOI : https://doi.org/10.7202/706627ar

Aller au sommaire du numéro

Éditeur(s)

École de service social de l'Université Laval

ISSN

1708-1734 (numérique)

Découvrir la revue

Citer ce document

Beaudoin, A. (1993). Antinomie ou concordance des perspectives théoriques en service social? Service social, 42(3), 3-5. https://doi.org/10.7202/706627ar d'utilisation que vous pouvez consulter en ligne.

https://apropos.erudit.org/fr/usagers/politique-dutilisation/ 


\section{AVANT.PROPOS}

\section{Antinomie ou concordance des perspectives théoriques en service social?}

Depuis les débuts du travail social, la question des fondements théoriques et scientifiques des pratiques a été au cœur des réflexions et souvent objet de débat. Au cours des dernières années, le débat a été partagé entre ceux qui pensent qu'il n'existe pas de théorie intrinsèque propre au travail social et ceux qui reconnaissent que la pratique même du travail social se fonde sur une théorie de l'action. Pour les premiers, parmi lesquels on trouve des administrateurs, des économistes, des politicologues, etc., le travail social est d'abord utilitaire et a des fondements normatifs et prescriptifs statutaires qui s'enracinent dans une société donnée. De ce point de vue, le travail social est surtout une pratique qui s'applique aux personnes et aux groupes dans la perspective de leurs droits sociaux. Pour les seconds, il y a un lien conceptuel entre théorie et action en ce sens que toute action peut être définie en lien avec une théorie de laquelle elle procède même si l'acteur n'en est pas toujours conscient. On débouche ainsi sur la possibilité de l'élaboration de cadres théoriques propres au service social.

Le lecteur va trouver dans les articles du présent numéro l'expression d'énoncés différents quant à des perspectives théoriques sur le travail social. À certains moments elles constitueront des antinomies les unes par rapport aux autres, mais à d'autres moments elles seront en concordance.

Dans le premier article, Jouthe nous incite à réfléchir sur une question fondamentale dans l'élaboration de toute théorie concernant le travail social: la question éthique. Partant d'un paradigme de l'unité d'identité 
et de la différence, il soutient qu'il est possible de négocier de façon coopérative les différences entre les individus, les groupes et les communautés. Dans cette négociation, les intervenantes et les intervenants sociaux prennent une place importante dans le cadre de l'équilibre à chercher ou à retrouver dans la société «pluraliste, multiraciale, multiethnique et multiculturelle» de cette fin de $\mathrm{XX}^{\mathrm{e}}$ siècle.

Les liens théoriques du service social à l'analyse politique sont l'objet de l'article de Gendron. Plus précisément, l'auteur fait ressortir les différentes conceptions du pouvoir et leur signification dans l'analyse du politique en lien avec l'évolution des institutions d'encadrement du service social. II montre en particulier comment la régulation des acteurs sociaux s'est d'abord manifestée par une technocratisation du travail social et l'exercice du pouvoir du service social au niveau microscopique plutôt qu'au niveau sociopolitique et socio-institutionnel plus global. Ainsi, à la suite de la mise en place des politiques sociales comme régulatrices de l'ensemble de la société, la relation de pouvoir est omniprésente dans le phénomène organisationnel qu'est l'intervention sociale.

Considérant la division entre la réalité de l'action professionnelle en "théorie » et "pratique», Zúñiga propose, pour sa part, une perspective constructiviste en service social pour combler les insuffisances des trois discours dominants (la "science", l'identification des "problèmes sociaux " et la «demande») dans le champ du travail social. Cette perspective qui situe la connaissance en tant que rapport social permet un nouveau discours de légitimation qui est source du développement $d^{\prime}$ 'une pensée autonome grâce à son orientation pédagogique et à son ouverture épistémologique et politique.

Après ces trois articles d'éclairage théorique d'ensemble, l'article collectif de Chamberland, Dallaire, Cameron, Fréchette, Hébert et Lindsay définit la prévention des problèmes sociaux et circonscrit les principales dimensions de la planification des actions préventives: les cibles et contextes de l'action; les moments de réalisation; les stratégies. Ce sont les fondements théoriques des différentes conceptions de l'action préventive qu'il faut en premier lieu retenir de cet article: modèle biomédical ; modèle de la santé communautaire; modèle écologique; contribution de la psychologie communautaire; et passage de la prévention des problèmes individuels aux problèmes communautaires dans une perspective $\mathrm{d}^{\prime}$ empowerment. Cela conduit les auteurs à proposer une définition préliminaire de la "prévention sociale».

Les deux contributions internationales au thème des "Perspectives théoriques » complètent le numéro. Elisabetta Neve fait une synthèse et présente les développements récents et les orientations actuelles de la 
théorie du service social en Italie. L'auteure concentre son analyse sur la redéfinition des orientations et des fondements de l'intervention professionnelle qu'on trouve en Italie en montrant la continuité des concepts de contrôle et d'aide et de la nécessité-possibilité de leur conjugaison à l'intérieur de l'intervention professionnelle. Cette redéfinition se transpose dans l'organisation institutionnelle des services et de la communauté territoriale à la base des interventions de réponse aux problèmes sociaux. En ce sens, la profession se nourrit de la réflexion «forte » qui conceptualise et définit l'action.

Quant à Jean-Marc Dutrénit, en partant d'une vision plus élargie et multi-professionnelle du travail social, il présente quatre grands paradigmes pour démêler l'apparent enchevêtrement des théories de la société, de la personne et de l'intervention sociale. Ces quatre paradigmes nés au cours du XXe siècle sont ceux du fonctionnalisme, de I'habitus culturel, de l'ethnométhodologie et de l'individualisme méthodologique. Après avoir analysé et critiqué les autres paradigmes, il retient celui de I'individualisme méthodologique, qui représente selon lui la meilleure contribution au développement de la réciprocité positive à la base de I'intervention sociale.

Comme on l'aura vu à la lecture de cet avant-propos, sans avoir répondu complètement à la question de départ, les articles de ce numéro présentent des perspectives théoriques qui contribuent à faire avancer la réflexion sur I'antinomie et la concordance entre les différentes perspectives de la réflexion théorique. 